\title{
Influence of laser parameters on laser ultrasonic efficiency
}

\author{
Andrew Forbes ${ }^{1 \#}$, Lourens Botha ${ }^{1}$, Neil du Preez ${ }^{2}$ and Tommy Drake ${ }^{3}$ \\ ${ }^{1}$ CSIR National Laser Centre, PO Box 395, Pretoria 0001, South Africa \\ ${ }^{2}$ Scientific Development and Integration (Pty) Ltd., PO Box 1559, Pretoria 0001, South Africa \\ ${ }^{3}$ Lockheed Martin Aeronautics Company, Lockheed Martin Blv, Fort Worth, Texas 76108, USA
}

\begin{abstract}
Laser ultrasonics is currently the optimal method for non-destructive testing of composite materials in the aerospace industry. The process is based on a laser-generated ultrasound wave which propagates inside the composite. The response at the material surface is detected and converted into a defect map across the aircraft. The design and optimisation of a laser system for this application is reviewed in this paper, together with the basic science involved. This includes the optimisation of laser parameters, such as output couplers and gas mixture, and the impact these choices have on the laser chemistry. We present a theory for the catalytic recombination of the gas which shows excellent agreement with experiment. Finally, an operating laser system for this application, yielding a six-fold improvement in performance over conventional laser systems, is described.
\end{abstract}

Keywords: Laser ultrasound, TEA $\mathrm{CO}_{2}$ lasers, laser chemistry, short pulses

\section{INTRODUCTION}

Polymer-matrix composites are increasingly used in the aerospace industry, particularly in the manufacture of modern fighter planes ${ }^{1-3}$. The number and complexity of such composites are also increasing steadily. The aerospace industry requires non-destructive testing (NDT) of all parts during the manufacturing process. In the case of composite materials, testing is particularly necessary for detecting the presence of delaminations and inclusions. The conventional approach involves the use of ultrasonics based on water jets and piezoelectric transducers. A requirement for this process is that the transducers must be normal to the surface to ensure good signal-to-noise ratio. Since most modern aircraft parts have a complex geometry, this process is slow and therefore expensive.

An alternative ultrasonic inspection technique is laser ultrasonics (LU). In this technique (see Figure 1) two lasers are used to illuminate the part: a short pulse laser with a wavelength chosen so as to be absorbed by the material, and a second laser beam to detect the impact of the ultrasonic waves. The first laser pulse is rapidly absorbed by the material, causing fast thermal expansion which results in ultrasonic waves. The wave amplitudes themselves are small enough not to cause any damage. The waves so generated propagate through the material and are reflected from interfaces (e.g., defects) inside the material. On returning to the surface, reflected waves generate very small surface displacements of the material. A second, narrow bandwidth, frequency stable laser is used in conjunction with an interferometer to detect these mechanical displacements on the surface of the material ${ }^{1-3}$. The advantage of LU is that these measurements can be done at large distances from the sources and at large angles with respect to the sample surface. This gives LU a competitive advantage and ensures that it is currently the NDT method of choice for the testing of composite materials in the aerospace industry.

For efficient and reliable operation, LU requires the efficient generation of ultrasound by the first laser pulse, which places stringent requirements on the laser system. The properties required of the laser pulse include good beam quality, a short duration time pulse $(<100 \mathrm{~ns})$, as well as a minimum energy $(>200 \mathrm{~mJ})$ and pulse repetition rate $(>200 \mathrm{~Hz})$ for the process to be suitable for industrial applications. Furthermore, since these systems will typically be used in a production environment, the final design must result in a laser system that is reliable (with low downtime) and economical. Numerical simulations and experimental investigations have indicated that generation efficiency can be improved by utilising short pulses in the $3-4 \mu \mathrm{m}$ and $10 \mu \mathrm{m}$ spectral regions ${ }^{1}$. Short pulse $10 \mu \mathrm{m}$ radiation can be produced by transversely excited, atmospheric $\mathrm{CO}_{2}\left(\mathrm{TEA} \mathrm{CO}_{2}\right)$ lasers. Due to the technological maturity of these lasers they are particularly suited to industrial environments.

\footnotetext{
\# Email: aforbes1@csir.co.za; ph: +27 12841 2368; fax: +27 128413152 


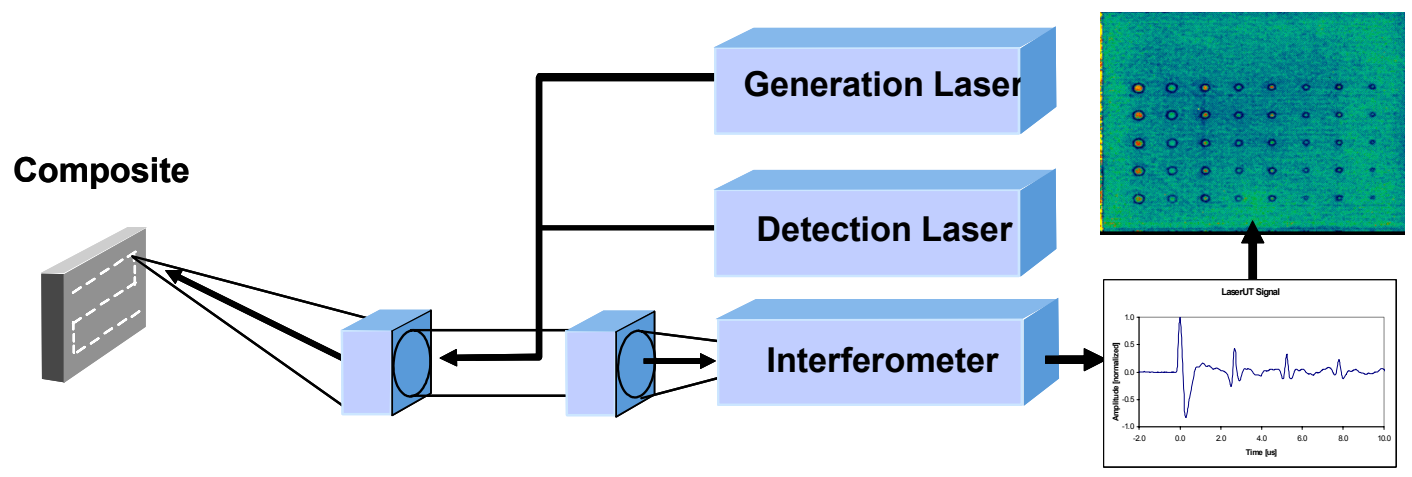

Figure 1: Illustration of the laser ultrasound principle.

In this paper we discuss the design of a TEA $\mathrm{CO}_{2}$ laser for $\mathrm{LU}$ applications. We cover the basic laser parameters to be optimised, and report experimental data for the optimisation of the output coupler reflectivity and gas mix. The impact on laser chemistry is discussed in detail.

\section{LASER PARAMETERS}

It is useful to start with a summary of the important laser parameters for LU, and their interrelationships. Since the parameters are not independent of one another, the critical aspect of the parameter testing is to explore regimes in terms of combinations of parameters. Only in some cases can the parameters be chosen independently. For LU a strong ultrasonic signal at as high a repetition rate as possible must be generated. The strong signal ensures a good signal-tonoise ratio, while a higher repetition rate allows more samples to be tested in a given time, thereby increasing productivity. The LU signal is a product of the efficiency of the laser pulse in generating ultrasound, determined by wavelength and temporal characteristics, and the energy of the laser pulse determined by gain/loss effects inside the resonator. A typical time pulse from a TEA $\mathrm{CO}_{2}$ is comprised of a short duration "spike" followed by a somewhat longer duration "tail". Since the total energy contained in the pulse is the time integrated signal, a long tail implies that most of the energy is contained in the latter part of the pulse. The time profile of the laser pulse is of paramount importance for efficient LU: one requires a very short time pulse $(<100 \mathrm{~ns})$, with as much of the energy in the spike as possible. Factors influencing the laser energy and time profile include gas mix, output coupler reflectivity (the amount of energy retained in the laser cavity), discharge volume and operating voltage amongst others.

Inevitably there are trade-offs. For example, high pulse energy from $\mathrm{TEA} \mathrm{CO}_{2}$ lasers is usually associated with high $\mathrm{N}_{2}$ content in the laser gas mix; but high $\mathrm{N}_{2}$ content is also associated with long time pulses. In general, the time envelope of the laser pulse is determined by the gain of the laser system. This in turn is largely influenced by both the reflectivity values of the cavity optics, and the gas mixture used in the laser. The gas mixture also has a direct influence (that is, not via the gain) on the time profile through the fraction of the excitation energy stored in the excited $\mathrm{N}_{2}$ upper energy levels. This energy is transferred to the upper energy levels of the $\mathrm{CO}_{2}$ molecule through collisions, resulting in a delayed energy output from the laser in the form of a characteristic "tail" observed in many pulses. Thus large $\mathrm{N}_{2}$ concentrations are usually associated with high energy and prolonged pulses with long tails. The general trend in the course of optimisation for shorter pulses, was towards higher $\mathrm{CO}_{2}$ fractions with lower $\mathrm{N}_{2}$ fractions. As the pulse time envelope and the tail are reduced, the total energy output drops. The energy drop was minimised through careful selection of the operating voltage (since the energy deposited into the laser discharge is proportional to the square of the operating voltage), and output coupler reflectivity. The problem was to find a working parameter set that maximised the LU signal, without adversely affecting the laser stability.

\subsection{Output coupler}

Four output couplers were used in experiments, with percentage reflectivities of $36 \%, 50 \%, 55 \%$ and $70 \%$. In addition to measuring the output energy as a function of OC, the LU signal was measured and the efficiency of the pulse in generating the signal was computed as a V/J value; that is, the amount of signal in Volts generated per Joule of input energy. These experiments were repeated for a range of gas mixtures (discussed in detail in the next section) in order to find the balance between high energy extraction and good LU efficiency, equivalent to short time pulse generation. 
Figure 2 shows a parametric plot of the LU efficiency (units of $\mathrm{V} / \mathrm{J}$ ) and measured output energies for the four tested OCs, with various high voltage (HV) settings (essentially various gain conditions) and gas mixtures. It is evident that, in general, lower OC reflectivity values give better LU efficiency at lower energies, indicating that such pulses have "better" pulse time characteristics for this application. As expected, a comparison of the pulse characteristics reveals that short pulses with small tails result in a better LU signal. The spread in results for a given OC is due to the gas mix and HV ranges used. Since the spread is in both efficiency and energy, one can deduce that these parameters also influence the time pulse characteristics.

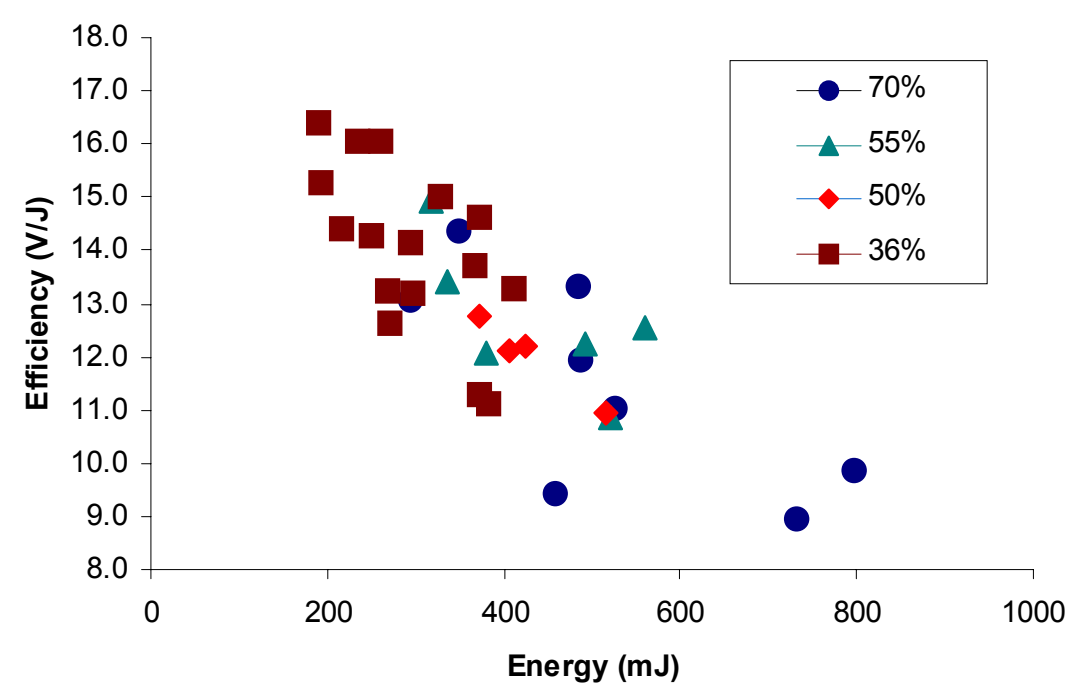

Figure 2: Laser ultrasound efficiency, measured as how many Volts of signal per Joule of laser energy. The scatter plot shows four OCs and a range of gas mixes.

In summary, if all other parameters are kept constant then increasing the output coupler reflectivity has a detrimental effect on the acoustic efficiency. The broad trend is for lower reflectivity OCs to outperform higher reflectivity OCs in terms of LU efficiency, while the reverse is true in terms of energy extraction from the laser. Ideally, therefore, one would like to use as low an output coupler reflectivity as is possible, and compensate for the energy loss in other ways, such as by adjusting the HV or optimising the gas mix used.

\subsection{Gas mixes}

Traditionally, a high energy TEA $\mathrm{CO}_{2}$ laser (with a closed gas system) has a He content of roughly $80 \%$ or more and a $\mathrm{CO}_{2}$ content of less than a few percent. The remainder would be mostly $\mathrm{N}_{2}$ with sometimes some $\mathrm{H}_{2}$ and $\mathrm{CO}$ to allow the gas to be "self-catalytic" (the $\mathrm{H}_{2}$ acts as a catalyst in the recombination of $\mathrm{CO}$ and $\mathrm{O}_{2}$ to form $\mathrm{CO}_{2}$ ). A $20 \% \mathrm{CO}_{2}$ fraction would be considered a very "rich" mix. Following the results shown in Figure 2, various gas mixtures were tested for energy extraction and time pulse characteristics, each with a different $\mathrm{He}$ and $\mathrm{CO}_{2}$ molecular fraction. Most tests involved mixes with less than or equal to $50 \% \mathrm{He}$ and high $\mathrm{CO}_{2}$ fractions. The general trend observed was towards shorter time pulses using higher $\mathrm{CO}_{2}$ fractions and lower $\mathrm{N}_{2}$ fractions. A pulse with excellent time characteristics for LU is shown in Figure 3. The pulse was measured using a gas with a $56 \% \mathrm{CO}_{2}$ fraction by volume. The resulting pulse had a full width, half-maximum (FWHM) time duration of $51 \mathrm{~ns}$, with no discernable tail and with more than $85 \%$ of the laser energy present in the first $100 \mathrm{~ns}$. Such a pulse shows a greater than 2-fold improvement in LU signal when compared to previously quoted values. However, lowering the $\mathrm{N}_{2}$ fraction decreased the total energy in the pulse. Increasing the HV compensated for the lower energy, but this in turn placed strain on the electrical components: the potential difference across the electrodes can increase to as much as $40 \mathrm{kV}$, placing strain on all electrical and insulating components. 


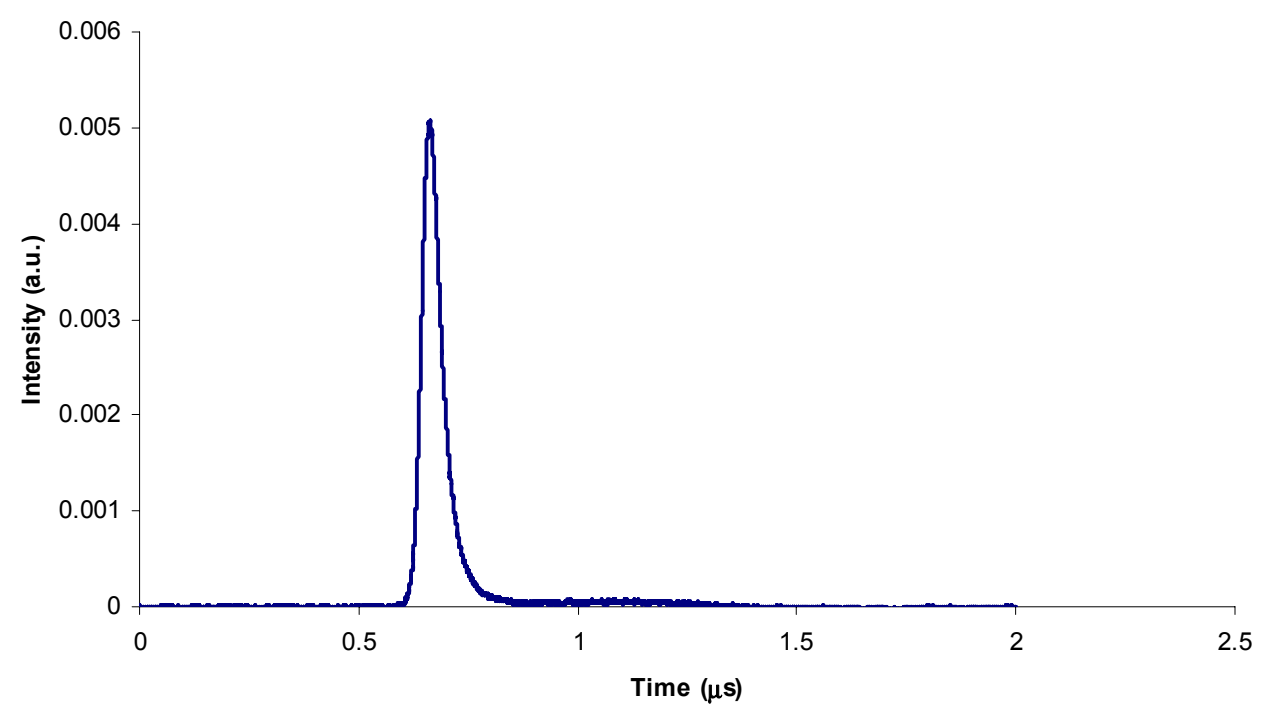

Figure 3: Example of a desired pulse for laser ultrasound - short spike, no tail, and more than $80 \%$ of the energy in the first $100 \mathrm{~ns}$.

\section{LASER CHEMISTRY}

The $\mathrm{CO}_{2}$ gas fraction in a TEA $\mathrm{CO}_{2}$ gas laser decomposes over time to mainly $\mathrm{CO}$ and $\mathrm{O}_{2}$ and a small amount of other by-products, as a result of the electrical discharge between two electrodes: $2 \mathrm{CO}_{2} \rightarrow 2 \mathrm{CO}+\mathrm{O}_{2}$. Thus, in the absence of other reactions, the $\mathrm{CO}_{2}$ concentration, $\left[\mathrm{CO}_{2}\right]$, decreases with time while the $\mathrm{O} 2$ and $\mathrm{CO}$ concentrations $\left(\left[\mathrm{O}_{2}\right]\right.$ and $[\mathrm{CO}]$ respectively) increase with time. This is a problem for a number of reasons. Firstly, as the $\left[\mathrm{CO}_{2}\right]$ decreases so the power output from the laser decreases. Secondly, as the $\left[\mathrm{O}_{2}\right]$ increases so the laser discharge becomes very unstable, with highly variable pulse-to-pulse energy values. Figure 4 shows a plot of laser pulse energy (total energy contained in each pulse) with oxygen concentration measured at simultaneous times $(400 \mathrm{~Hz}$ measurements - i.e., at the repetition rate of the laser). We have verified (not reported here) that the energy decrease is due to the $\left[\mathrm{CO}_{2}\right]$ decrease. Additionally, there is a marked increase in the pulse to pulse energy variability at high oxygen concentration levels. We attribute this to the adverse effects of $\mathrm{O}_{2}$ in such electrical discharges which often lead to electrical arcing.

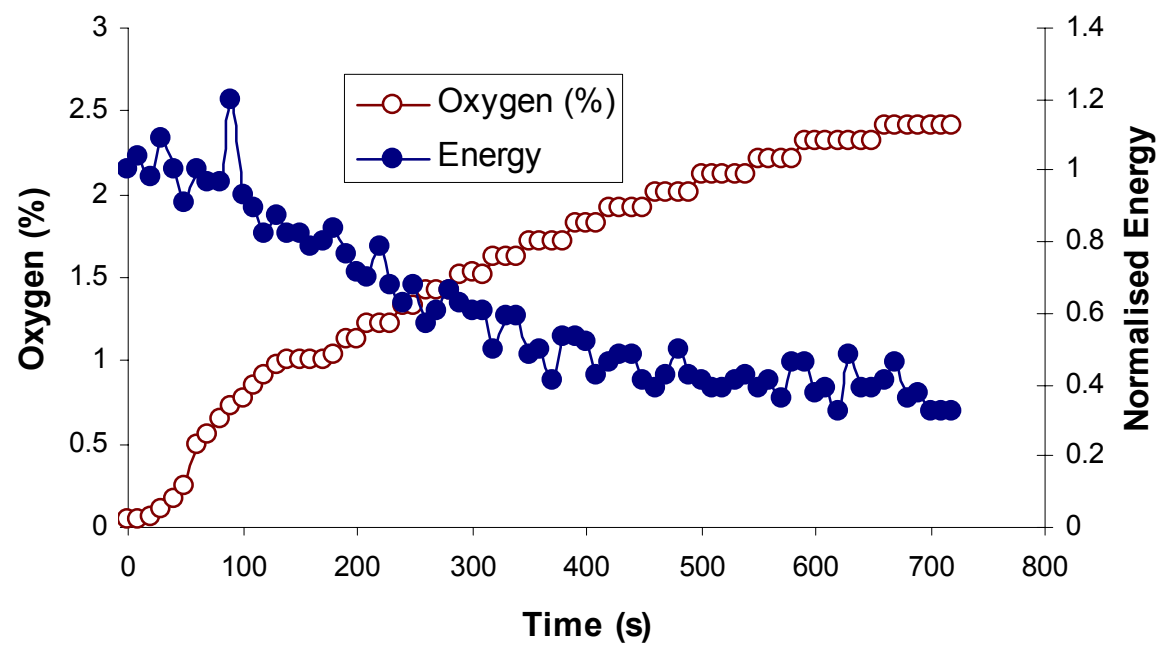

Figure 4: The oxygen concentration increases due to the dissociation of $\mathrm{CO}_{2}$. $\mathrm{Less}_{\mathrm{CO}}$ results in less energy output from the laser. 
Additional experiments suggest that the increase in [CO] does not have any deleterious effects on the laser power output. Thus the decomposition of $\mathrm{CO}_{2}$ contributes both to a decrease in laser energy and an increase in laser instability. Needless to say, both these effects need to be minimised.

The laser was operated with internal, room temperature catalysts, mounted downstream of the gas flow through the discharge volume but just upstream of the heat exchanger. Thus the hot depleted gas first passed through the catalysts for regeneration, and then through the heat exchanger for cooling. In an ideal system, a single round trip would regenerate the initial gas conditions, a cool gas still rich in $\mathrm{CO}_{2}$, so that the process repeats endlessly. The role of the catalysts is to approximate this state by reversing the breakdown reaction through the oxidation of $\mathrm{CO}^{\text {back }}$ to $\mathrm{CO}_{2}$. In the case of room temperature catalysts (usually $\mathrm{Pt} / \mathrm{Sn}$ based), the mechanism is not well understood. Some studies ${ }^{4}$ indicate that excess $\mathrm{CO}$ improves catalyst performance, as well as reducing $\mathrm{O}_{2}$ formation in the discharge, while other studies $^{5,6}$ show the converse. It seems generally agreed ${ }^{4-7}$ that excess $\mathrm{O}_{2}$ is undesirable for both laser action and catalyst activity. In the case of a $\mathrm{Sn}-\mathrm{Pt} / \mathrm{SiO}_{2}$ catalyst, the presence of pure $\mathrm{O}_{2}$ for a period of 15 minutes resulted in complete loss of catalyst activity ${ }^{5}$.

We have previously ${ }^{8}$ presented details of a model for the change in oxygen concentration inside the laser. A simple rate law was found to accurately predict the oxygen concentrations over time, given by:

$$
x(t)=\left(x_{0}-\frac{k_{d}}{k_{g}+k_{c}}\right) \exp \left(-\left(k_{g}+k_{c}\right)\left(t-t_{0}\right)\right)+\frac{k_{d}}{k_{g}+k_{c}} .
$$

Here $x(t)$ is the oxygen concentration as a function of time, $k_{g}, k_{c}$ and $k_{d}$ are the rate constants associated with (a) the gas recombination at high temperature, (b) the catalysts and (c) the discharge respectively. It is assumed that the system starts with some oxygen concentration $x_{o}$ at time $t_{o}$. The model was then tested against an arbitrary initial condition of a running laser, followed by a period when the laser is switched off (no discharge), and then switched on again (discharge on) and allowed to stabilise. The test is comprehensive because it requires the model to describe both discharge and nondischarge conditions, as well as both transient and steady state periods of operation. The success of the model is shown in Figure 5, where the model prediction (solid curve) shows excellent agreement with the experimental data (data points). Similar agreement is found between experiment and theory when no catalysts are present.

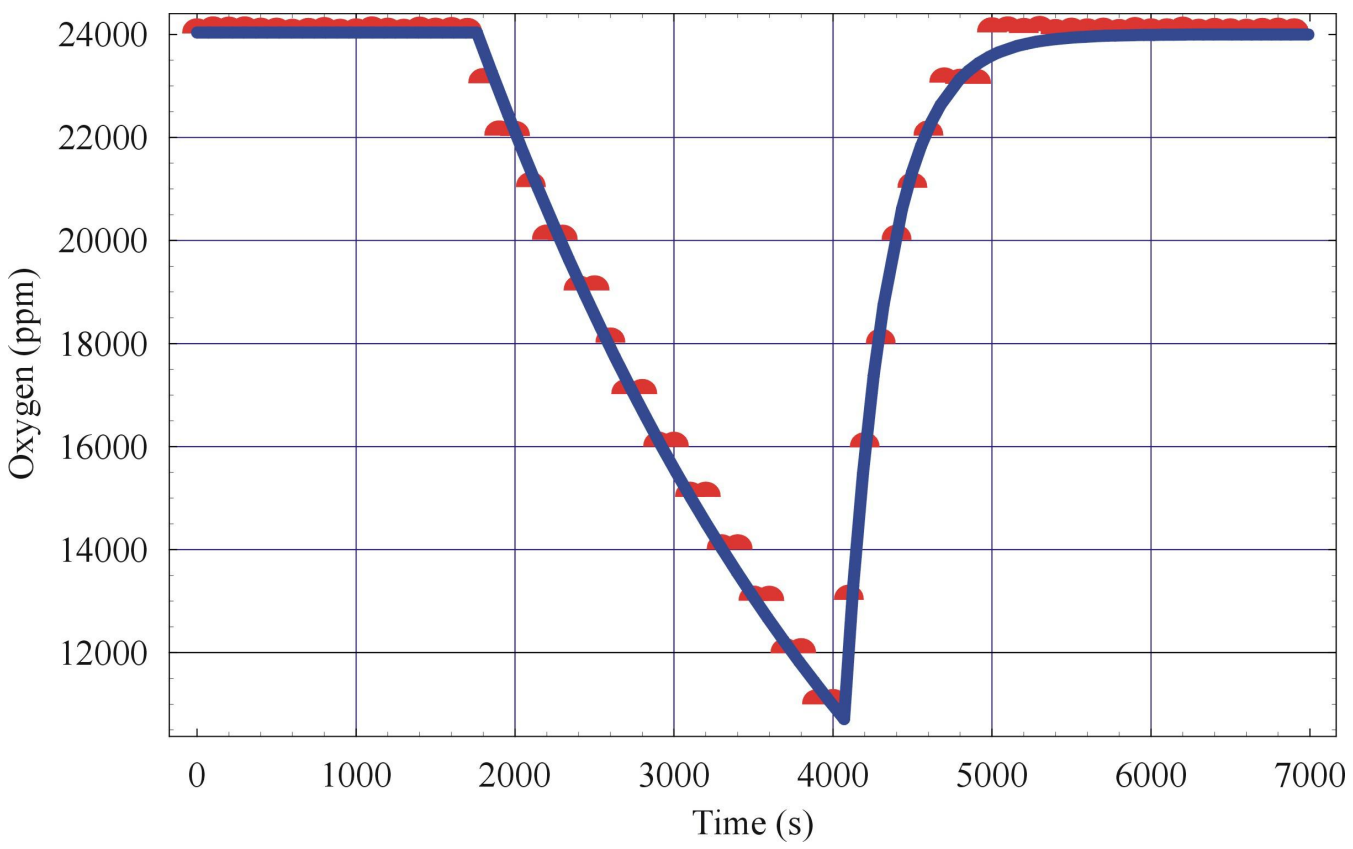

Figure 5: Agreement between model (solid curve) and experimental data. 
One can use the model to make certain predictions as to the behaviour of the gas in the presence of catalysts. Although it is useful to know the time evolution of the oxygen concentration, as given by Equation (1), one is usually interested in predicting the final steady state concentrations of oxygen in the system, since this is the level at which the laser will be expected to operate at for extended time periods. If the value is too high, the laser will be unstable in operation (e.g., due to a high arc rate or a low output energy). One can also use the model to investigate what initial conditions are necessary to ensure stable operating regimes. Allowing $t \rightarrow \infty$ in Equation (1), one finds the predicted oxygen levels at steady state as

$$
\left[O_{2}\right]_{e q}=\frac{k_{d}}{k_{g}+k_{c}} \approx \frac{19 P_{i n}}{k_{c}} .
$$

Here $P_{\text {in }}$ must be expressed in $\mathrm{kW}$, and the factor 19 has been determined empirically ${ }^{8}$ by running the laser discharge without any catalysts and monitoring the oxygen increase as a function of power input. From this equation we see that good quality catalysts, with high $k_{c}$ values, will lead to a reduction in the final oxygen concentration at steady state. We have shown before that increasing the power does not necessarily lead to an increase in the oxygen concentration levels at steady $\operatorname{state}^{8}$.

\section{CONCLUSION}

We have completed a design and optimisation process on a TEA $\mathrm{CO}_{2}$ laser to be used in LU. The results show that for LU applications, lower output coupler reflectivities and gas mixes with high $\mathrm{CO}_{2}$ content are preferred. The process included a detailed study of the chemistry within the laser, and the impact this has on laser performance over time.

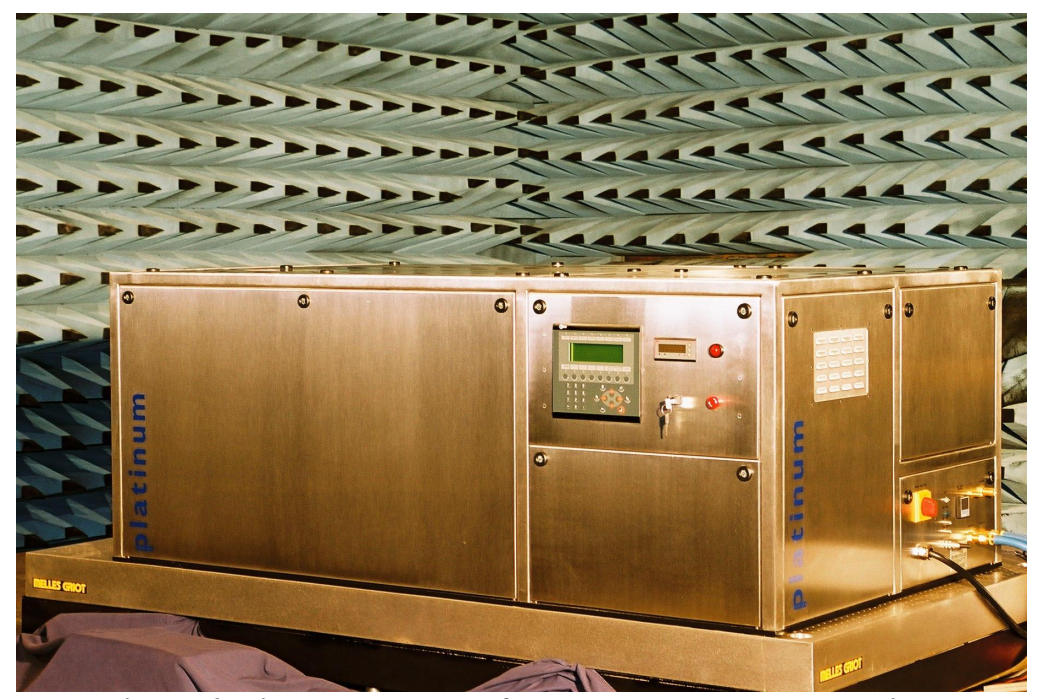

Figure 6: Final laser system for laser ultrasound generation.

The outcome of the study was a final laser parameter configuration suitable for use in LU. The final laser design is presently under manufacture at Scientific Development and Integration, a South African technology company, where contract research and development continues. The prototype system discussed in this paper is in operation at a Lockheed Martin aerospace company for LU testing of modern jet fighters. An example of such a system is shown in Figure 6. 


\section{REFERENCES}

1. Dubois M., Chuang S.Y., Lorraine P.W., Drake T.E., Yawn K.R. and Filkins R.J. 2001. Progress on the Development of an advanced laser ultrasound generation source for inspecting polymer-matrix composites. Review of Progress in Quantitative Nondestructive Evaluation 21A, pp. 300-308.

2. Scruby C.B. and Drain L.E. 1990. Laser-ultrasonics: Techniques and applications. Taylor and Francis, New York, pp. 1-462.

3. Monchalin J.-P., Néron C., Bussiere J.F., Bouchard P., Padioleau C., Hénon R., Choquet M., Aussel J.-D., Carnois C., Roy P., Durou G. and Nilson J. 1995. Laser ultrasonics: from the laboratory to the shop floor. Physics in Canada 51, pp. 122-130.

4. Lewis P.F. and Tammaro E. 1999. Recombination Catalysts for Pulsed Self-Sustained $\mathrm{CO}_{2}$ Electrical Discharge Lasers. In Proceedings of the International Conference on Lasers, Quebec, Canada, ed. V.A. M'Lean and V.J. Corcoran, pp. 351-358.

5. Margitfalvi J.L. 2002. Low Temperature oxidation of $\mathrm{CO}$ over tin-modified $\mathrm{Pt} / \mathrm{SiO}_{2}$ catalysts. Catal. Today 73, pp. 343-353.

6. Schryer D.R. and Upchurch B.T. 1990. Effects of pre-treatment conditions on a $\mathrm{Pt} / \mathrm{SnO}_{2}$ catalyst for the oxidation of $\mathrm{CO}$ in $\mathrm{CO}_{2}$ lasers. J. Catal. 122, pp. 193-197.

7. Marchetti R., Penco E., Salvetti G. 1985. Sealed, Miniaturised, Corona-Preionised, High-Repetition-Rate TEA CO Laser Using Hydrogen Buffered Gas Mixtures. IEEE. J. Quantum Electron. QE-21, pp. 1766-1771.

8. Forbes A. and Botha L.R. 2005. Predicting gas decomposition in an industrialised pulsed $\mathrm{CO}_{2}$ laser. In Proceedings, XV International Symposium on Gas Flow, Chemical Lasers and High Power Lasers, Prague, Czech Republic, ed. J. Kodymova, pp. 491-494. 